University of Wollongong

Research Online

Faculty of Engineering and Information

Faculty of Engineering and Information

Sciences - Papers: Part A

Sciences

January 2015

\title{
Enhancing teamwork performance in mobile cloud-based learning
}

Geng Sun

University of Wollongong, gs147@uowmail.edu.au

Jun Shen

University of Wollongong, jshen@uow.edu.au

Follow this and additional works at: https://ro.uow.edu.au/eispapers

Research Online is the open access institutional repository for the University of Wollongong. For further information contact the UOW Library: research-pubs@uow.edu.au 


\title{
Enhancing teamwork performance in mobile cloud-based learning
}

\begin{abstract}
Mobile cloud-based learning is a novel trend that allows collaborative learning to happen among distributed learners, but it still lacks of mechanisms to enhance teamwork performance. Combining the features of the cloud, we have identified a learning flow based on Kolb team learning experience, executed by cloud-hosting learning management systems in conjunction with our newly designed system, 'Teamwork as a Service (TaaS)'. Each of TaaS's five web services aims to organize a certain type of learning activities, providing learners with an introduction, a 'jigsaw classroom', schedule planning, and mutual supervision during the whole collaborative learning process. In particular, enabling a rational group mechanism realized by the simulated annealing method, TaaS is able to allocate learners to their appropriate tasks in order to give their best performance. We also introduce details of the implementation of TaaS over the Amazon cloud.
\end{abstract}

\section{Keywords}

performance, mobile, cloud, learning, teamwork, enhancing

\section{Publication Details}

Sun, G. \& Shen, J. (2015). Enhancing teamwork performance in mobile cloud-based learning. Lecture Notes in Computer Science, 8390 107-117. 


\title{
Enhancing Teamwork Performance in Mobile Cloud- Based Learning
}

\author{
Geng Sun, Jun Shen \\ School of Information Systems and Technology \\ University of Wollongong \\ Wollongong, 2522, NSW, Australia \\ gs147@uowmail.edu.au, jshen@uow.edu.au
}

\begin{abstract}
Mobile cloud-based learning is a novel trend that allows collaborative learning to happen among distributed learners, but it still lacks of mechanisms to enhance teamwork performance. Combining the features of the cloud, we have identified a learning flow based on Kolb team learning experience, executed by cloud-hosting learning management systems in conjunction with our newly designed system, 'Teamwork as a Service (TaaS)'. Each of TaaS's five web services aims to organize a certain type of learning activities, providing learners with an introduction, a 'jigsaw classroom', schedule planning, and mutual supervision during the whole collaborative learning process. In particular, enabling a rational group mechanism realized by the simulated annealing method, TaaS is able to allocate learners to their appropriate tasks in order to give their best performance. We also introduce details of the implementation of TaaS over the Amazon cloud.
\end{abstract}

Keywords: mobile learning, cloud computing, collaborative learning, teamwork performance enhancement

\section{Introduction}

Mobile learning (m-learning) is an evolved type of electronic learning (e-learning), which is very useful for learners, enabling them to learn wherever they are and whenever they want. It is obvious, however, that mobile devices are limited by insufficient computing speeds, lower storage space and narrower screen size. To make up for such shortcomings, linking m-learning to cloud computing in order to borrow powers supported by the cloud is a novel solution in which mobile devices are only used for input and output of data. Cloud computing provides massive data-handling capability, elastic storage, on-demand service and faster processing speed in order to facilitate $\mathrm{m}$ learning, and, in addition, prompt and large-scale deployments of learning management systems (LMSs) is also easily enabled [21]. Hence, the application of mobile cloud-based learning is gaining wide acceptance [13].

Many system developers and researchers are interested in drawing support from cloud computing to build virtual learning environments (VLE) for m-learning, adopting the concept of service-oriented architecture (SOA) [5]. In addition, making use of

adfa, p. 1, 2011.

(C) Springer-Verlag Berlin Heidelberg 2011 
Web 2.0 technologies, several of the latest LMSs are able to offer collaborative learning tools [22]. Because they are hosted over the cloud and available for mobile access, it is possible for multiple learners to work together towards a common target by using mobile cloud-based learning.

On the other hand, to our knowledge, although collaborative learning happens more and more frequently in the mobile cloud-based learning environment, there are still comparatively few studies focusing on enhancing teamwork performance in this novel environment. In this paper, we introduce a novel approach to fill this gap in research, by offering a service-oriented system, 'Teamwork as a Service' (TaaS), which works as a third-party system by adding teamwork-focused functions to current cloud-hosting LMSs.

\section{Motivation and Methodology}

The context of mobile cloud-based learning is more specific than traditional learning, where learners are distributed over large geographical areas, even in countries all around the world. These virtual teams are more focused on task-related outcomes and time constraints [6]. Thus, once a teamwork assignment is given in an m-learning course, because of geographical separation and even time differences, learners are faced with many unpredictable difficulties for which they are not prepared and perhaps the biggest of these is insufficient communication [15].

In addition, there are problems which also occur in traditional team learning which can negatively affect mobile team learning. The literature shows that learners belonging to the same team often have differing learning styles and therefore require diverse learning approaches [7]. Each learner's expectations and preferences also influence their motivation to work to the limit of their abilities [18].Current assessment criteria also lack the mechanism to track the entire learning experience, and are generally based on learners' final outcomes. This means that problems can be hard to diagnose and solve in a timely manner, while the team learning is actually in progress.

Another character of m-learning is that its learning activities normally consist of two sections: online learning and offline learning [20]. Because mobile learners are free to download material into their mobile devices for viewing offline and being introduced and guided into their practices, they do not always stay online to access LMSs and attend tutorials [2]. A new concept, 'online to offline' (O2O), can help organize mobile cloud-based learning [24]. Using this, the process logic of mobile team learning can be clearly defined by online systems, including the transaction details and deliverable resources. So while learners are able to accomplish many of their teamwork tasks offline, for some necessary procedures, such as data entry and work submission, they need to go back online to finish.

To utilize the $\mathrm{O} 2 \mathrm{O}$ concept to facilitate collaborative learning in the mobile cloud environment, we need to consider several aspects in order to exploit the merits of online systems:

- The system should be service-oriented to support flexible interoperation, especially with current LMSs. 
- The interfaces of the system should be user-friendly when accessed through mobile devices.

- The Learners' strengths and weakness with regard to their learning styles should be identified [19].

- The learning process should be concise with indispensable activities, and more importantly, enable rational grouping within [16].

A feasible way to realize this teamwork-enhanced learning process is to orchestrate a learning flow [4], by compositing several web services. Generally, the traditional collaborative learning flow in mobile cloud-based learning can be abstracted as "receiving team assignments", "accessing team learning resources", "proceeding team learning" "submitting team outcomes" and "getting evaluations". By using Kolb's 'team learning experience' (KTLE) as the main concept [8], we implemented a teamwork-enhanced learning flow by automatically interoperating cloud-hosting LMSs and our newly designed service-oriented system, TaaS, that emphasizes building a better context for team learning. In the novel learning flow, which is shown as Figure 1 , the "proceeding learning content activities" is subdivided into the seven modules of KTLE, one or more of which are taken by each of the five web services of TaaS to organize a certain type of learning activity, working in parallel with the activity of "accessing learning resource".

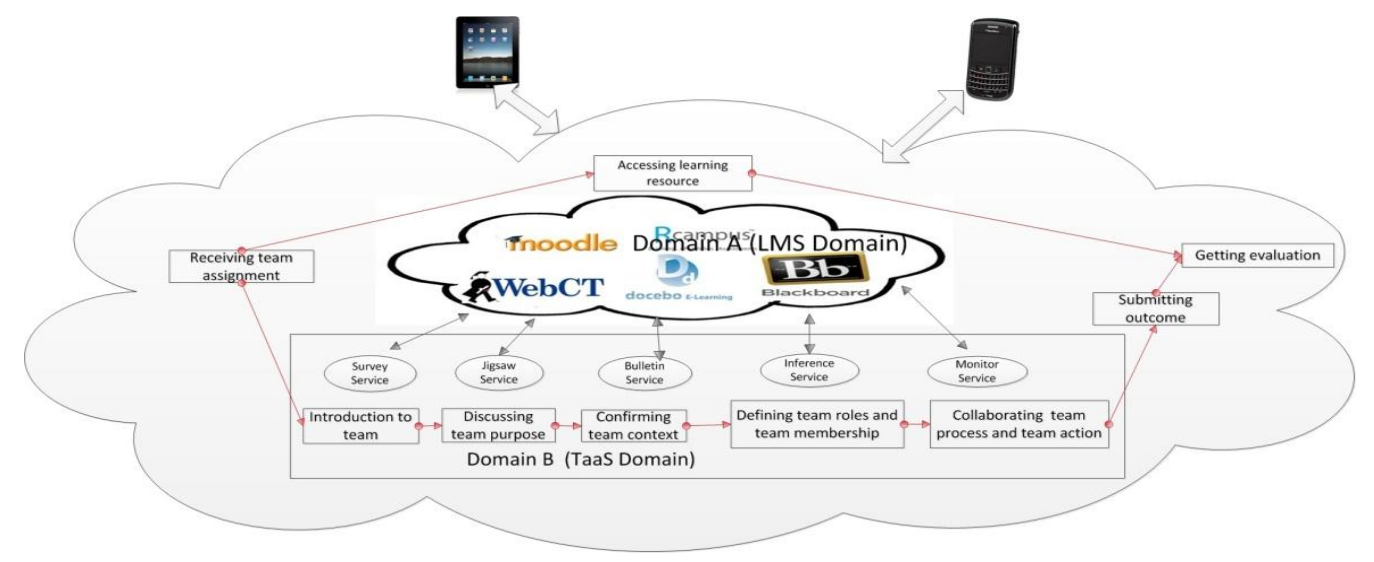

Fig. 1. Teamwork-Enhanced Learning Flow for Mobile Cloud-Based Learning

\section{System Framework}

\section{1 "Introduction to Teams" The Survey Service}

The Survey Service is used for gathering data of learner information [19], which is about the Kolb's Learning Style (KLS) and their comprehensive teamwork skills. It offers interfaces to learners for answering questions to investigate their capabilities. Considering the limitation of screen sizes and typing method of the mobile devices, the survey is single-choice based. The survey can be operated as self-assessment or 
peer-assessment, which means the respondents of the surveys, can evaluate themselves or the other group members working with them by giving appropriate marks.

There are five sets of questions being pre-installed in the Survey Service, four of which are for the four aspects of KLS [9][10], and the last is for comprehensive teamwork skills. These questionnaires come from [11] [23], and can be extended or reduced by teachers manually.

\section{2 "Team purpose" The Jigsaw Service}

The jigsaw method introduced in [1] is classic for organizing efficient discussion about "team purpose" among learners, the three stages of which can be imitated by the Jigsaw Service:

1. For "initial discussion in original team", the Jigsaw Service groups learners into four-person original teams, keeping the total comprehensive teamwork skills of each equal with the others'. In each original team, the four KLS team roles are separately assigned to members [3].

2. For "joining expert team to refine cognition", it rebuilds four expert teams, within each of which learners who played the same roles in the original teams are involved.

3. For "backing to original group to teach others what was gained in expert group", it redirects learners into the original teams from which they have come.

\section{3 "Team Context" The Bulletin Service}

The Bulletin Service provides a platform for learners to collaboratively define the "team context" and on which they are able to publish schedules of alternative tasks, each of which is suitable for an imaginary team and consists of several subtasks. The publisher of a task is required to mark the difficulty of its subtasks as expectedachievable values in KLS, while other learners are free to show their preferences regarding those when browsing. As it is in WYSIWYG mode, publishing the task schedule through user interface is easily done. In addition, subtasks' difficulty and learners' preferences are also marked using a multiple-choice format.

\section{4 “Team Membership” and "Team Roles" The Inference Service}

For "team role" and "team membership", the Inference Service works like a team leader. Referring the capabilities and the preferences of learners, and the expectedachievable values of subtasks, it assigns each learner a subtask, and also groups learners who take subtasks belonging to the same task into a team. This is the core of TaaS because it makes rational decisions to cover the uncertainty of the mobile environment, concentrating on outlining learners' responsibilities clearly and bringing their strengths into full play. We suppose two ways of forming a team, with different focus:

- "Keeping the balance between each team", which means the upcoming teams will have approximate comprehensive teamwork skills. In addition, the learners' pref- 
erences and capability levels are diverse in confined shapes, meaning that if we regard each team as an independent unit, its integrated preferences and capability values are highly close to those of other units. Therefore, we can say that the interteam competition between the upcoming teams starts from the same scratch line and is assured fair.

- "Letting the learners show their capabilities mostly", which means each of them is able to put their superiorities to use as much as possible, so that whether the team members are "good at" and "happy to" their upcoming subtasks will be the main indexes that direct the reasoning processing of task allocation.

The mathematical model of the task allocation is shown in Table 1.In [17], we introduce a simulated annealing (SA) method to solve the problem of task allocation. The operation of SA is to use simulated annealing regulation to optimize a set of initial solutions, iteration by iteration, towards an ultimate purpose for each, which is described by an objective function. The initial solution is a random array of $k$ learner/subtask pairs, where $k$ is the number of learners. A new solution is generated by swapping the positions of learners of two learner/subtask pairs in a previous solution.

Table 1. Problem Setting and Definition for Computing in The Inference Service

\begin{tabular}{|c|c|c|}
\hline Element & Definition & Notation \\
\hline$L^{k}$ & The $\mathrm{k}^{\text {th }}$ Learner & Learner ID \\
\hline Task $^{i}$ & The $\mathrm{i}^{\text {th }}$ Task & Task ID \\
\hline Suntask $^{i j}$ & The $\mathrm{j}^{\text {th }}$ Subtask of the $\mathrm{i}^{\text {th }}$ Task & Subtask ID \\
\hline$K L S^{k}$ & $L^{k}$ 's survey results of KLS capability & $\begin{array}{l}K L S^{k}=\left\{A C^{k}, A S^{k}, C^{k}, D^{k}\right\} \text {, each value is a real between } 1 \\
\text { and 10. } A C^{k}, A S^{k}, C^{k}, D^{k} \text { and } C T^{k} \text { represent the capability } \\
\text { values of accommodating, assimilating, converging, } \\
\text { diverging and comprehensive teamwork skills, respective- } \\
\text { ly. Note the first four values are according to KLS. }\end{array}$ \\
\hline$C T^{k}$ & $\begin{array}{l}L^{k} \text { 's survey results of comprehensive } \\
\text { teamwork capability }\end{array}$ & $C T^{k}$ is a real between 1 and 10 \\
\hline$P_{k}^{i j}$ & $L^{k}$ 's preference to the Suntask $k^{i j}$ & $P_{k}^{i j}$ is an integer between 1 and 5. \\
\hline$S T^{i j}$ & $\begin{array}{l}\text { Suntask }^{i j} \text {, s expected-achievable } \\
\text { degree in KLS }\end{array}$ & $\begin{array}{l}S T^{i j}=\left\{A C^{i j}, A S^{i j}, C^{i j}, D^{i j}\right\}, \text { each value is a real between } 1 \\
\text { and } 10\end{array}$ \\
\hline$N^{i}$ & The number of subtask in the $i^{\text {th }}$ Task & $N^{i}$ is a integer greater than 0 \\
\hline$D e P_{k}^{i j}$ & $\begin{array}{l}\text { The preference gap between } L^{k} \text {, s } \\
\text { ideal and reality. }\end{array}$ & $D e P_{k}^{i j}=5-P_{k}^{i j}$ \\
\hline$D e K_{k}^{i j}$ & $\begin{array}{l}\text { The deviation of } L^{k} \text { 's KLS capability } \\
\text { values versus Suntask }{ }^{i j} \text { s KLS ex- } \\
\text { pected-achievable degree. } \\
\text { (Note the deviation is defined by the } \\
\text { expression of Euclidean distance. It } \\
\text { is the lower the better.) }\end{array}$ & $\begin{array}{l}D e K_{k}^{i j}=-\left\{\operatorname{sign}\left[\sum\left(K L S^{k}-S T^{i j}\right)\right]\right\} \cdot\left\|K L S^{k}-S T^{i j}\right\| \\
\text { subject to: } \\
K L S^{k}-S T^{i j}=\left\{A C^{k}-A C^{i j}, A S^{k}-A S^{i j}, C^{k}-C^{i j}, D^{k}-D^{i j}\right\} \\
\left\|K L S^{k}-S T^{i j}\right\|=\sqrt{\left(A C^{k}-A C^{i j}\right)^{2}+\left(A S^{k}-A S^{i j}\right)^{2}+\left(C^{k}-C^{i j}\right)^{2}}\end{array}$ \\
\hline
\end{tabular}

Solutions in the two scenarios should be measured by (1) or (2), respectively. The ultimate solution will not be reached until achieving the lowest value of $R_{m}$ : 


$$
\begin{gathered}
\mathrm{R}_{\mathrm{m}}=\operatorname{Min}(\alpha \operatorname{Var} C T+\beta \operatorname{VarDeP}+\gamma \operatorname{VarDeK}+\varepsilon \overline{D e P}+\eta \overline{D e K}) \\
\mathrm{R}_{\mathrm{m}}=\operatorname{Min}(\alpha \operatorname{Var} C T+\beta \operatorname{SumDeP}+\gamma \operatorname{SumDeK})
\end{gathered}
$$

\section{5 "Team Process" and "Team Action" The Monitor Service}

The Monitor Service aims to provide mutual supervision for "team process" and "team action". In each team, it appoints one learner as the coordinator for each subtask, who is different from the task completer [14]. Each pair of them is linked by a file transmission channel, through which the completer is asked to submit their periodical outcome to be reviewed. The coordinator takes responsibilities to judge whether his corresponding completer has reached the rate of progress and are capable to continue or not, by grading him "satisfactory" or "unsatisfactory". A penalty mechanism is embedded in this service. It automatically deducts the completer's marks if he gets any "unsatisfactory" grade on a stage of his work in progress. All lost marks are accumulated and fed back to teachers at the end of team learning.

\section{System Implementation}

To implement TaaS, we have launched a Linux instance, of the Amazon Elastic Cloud Computing (EC2). We have configured the server environment as Apache + PHP + Mysql, and hosted our TaaS package on it. We have uploaded an open-source LMS, MOODLE, into the Amazon EC2, hosted on the same instance.
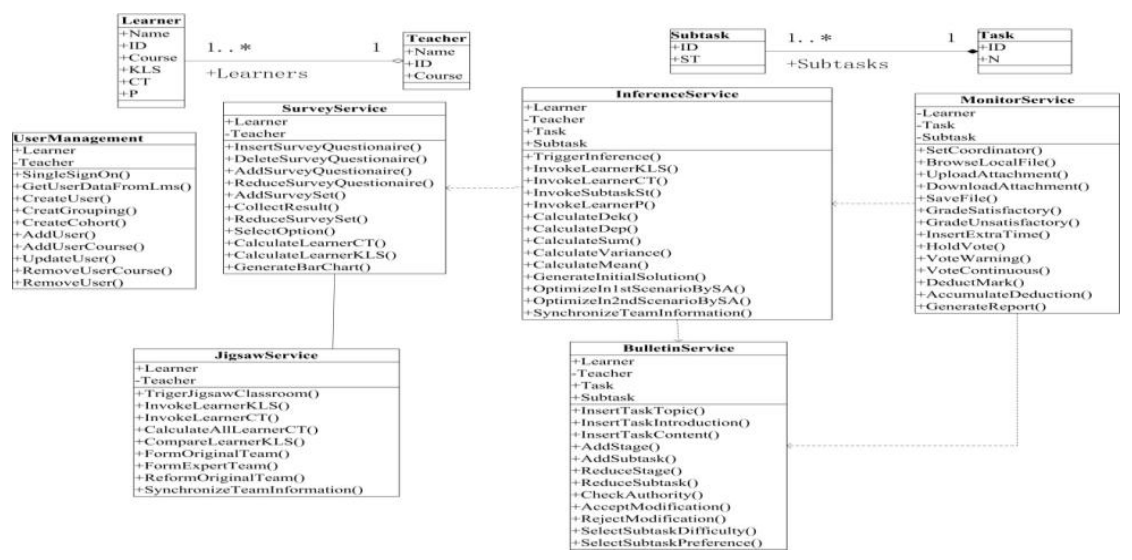

Fig. 2. UML Class Diagram of TaaS

As shown in Figure 2, these services are integrated in a system, and we have added a user-management module for controlling accounts. Abbreviations in this class diagram can be referred to the Table 1 , and the web methods provided by each service are also listed. The single-sign-on (SSO) technique is realized to enable users (teach- 
ers and learners) to log in to TaaS if they have valid MOODLE accounts. We created a new database of TaaS for storing teamwork-related data, such as learners' KLS capabilities, preferences, etc, meanwhile basic learning information, such as learner name, course name, etc, are invoked from MOODLE through its web service APIs, namely, core_user and core_course. In addition, the new database exposes a web service API for remotely invoking from LMSs other than MOODLE. After any change of team information, TaaS automatically updates it to MOODLE by invoking the core_group API.

The screenshots of UI are caught from a Samsung Tablet. Users are free to access TaaS and cloud-hosting LMSs by simple operation (e.g. finger actions on the touch screen) through their mobile devices, while the whole computing process is handled over the cloud. The UI of teachers' main page of TaaS is shown as Figure 3. Teachers can click buttons to launch several events, such as starting each stage of the Jigsaw classroom and activating grouping by triggering the Inference Service. They also have authority to change the structure of surveys, pre-set the deduction for the learner's each "unsatisfactory" outcome, the number of subtasks in a task and so on.

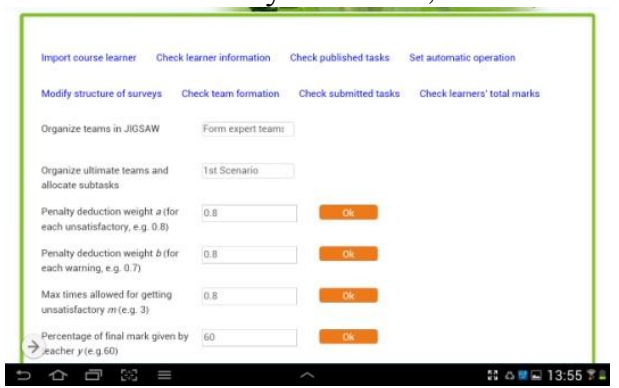

Fig. 3. Main Page of the Teacher User

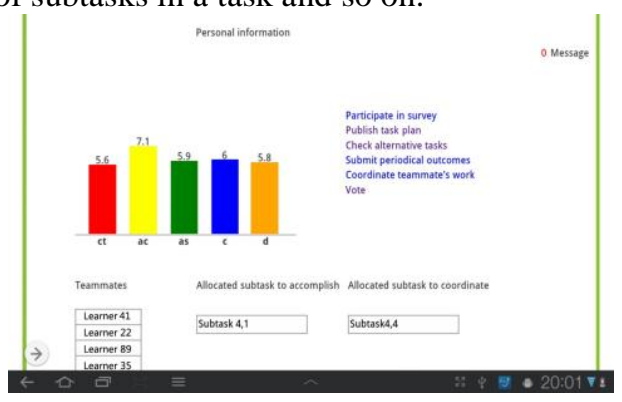

Fig. 4. Main Page of the Learner User

The UI of learners' main page is shown as Figure 4. Learners' capabilities in five areas are summarized in a bar chart, and can be checked by their teammates. They can click buttons to participate in learning activities by entering new pages. The status of the message box changes when the new announcement arrives. Their team information and task information are shown on the bottom of the main page. While they are planning schedules using the Bulletin Service, the structure of tasks is scalable, by adding/reducing subtasks and adding/reducing the stages of subtasks.

\section{Discussion}

On-demand service is a prominent feature of cloud computing. Thanks to web services in the cloud environment being loosely coupled, the architecture of serviceoriented systems is flexible. TaaS is therefore customizable depending on the teaching plan in mobile cloud-based learning, which means parts of these five web services can be de-coupled or re-coupled to work individually to meet special requirements.

In any case, the use of the integrated system is recommended for enhancing teamwork performance. In many cases in the mobile environment, learners' behaviors and 
mental abilities vary greatly, while teamwork is more related to human-to-human interaction rather than human-to-machine interaction. Even though collaborative learning tools are not rare in the current Internet environment and the use of social networking is improving the convenience of digital communication, the learning activities of virtual teams are still difficult to maintain, because of such problems as incompatibilities between different learners' abilities and learning styles. Thus, it is useful for an online system to contribute to the guidance and regulation of what learners do offline, so as to maintain progress towards their common goals. Additionally, as TaaS exposes standardized service-oriented APIs that allow dynamic integration over the web, they can be easily invoked by external services and are seamless to work in conjunction with LMSs for building a function-complete VLE.

Hosting TaaS over the cloud can enable the multiple accesses from education providers in different level by one large-scale deployment of TaaS, and let TaaS be protected by load balancers in the cloud to keep the robustness when suddenly increasing visit volumes occur. The needs for data and computation during the team learning process can be controlled by the cloud, thus the complexity of system will not be aggravated by the limitations of the mobile devices. Taas has the ability to solve problems which could undermine the work of the whole team. Main enhancements of teamwork performance brought by TaaS are the following:

- The mature KTLE theory helps learners to structure the essential competencies necessary for team learning in a succinct way, which can be executed smoothly using mobile devices.

- Learning styles are identified by means of KLS, in order to explore learners' strengths. It aims to improve efficiency by ensuring that the completer is the 'expert' in the subtask she/he is entrusted with. For example, a learner who is better at active experimentation and concrete experience is appropriate to be allocated a subtask of "accommodating", whereas a subtask of "assimilating" suits a learner who has stronger skills of abstract conceptualization and reflective observation.

- Knowing one another is very useful to help teammates to prepare for their following work. However, in the mobile learning environment, learners find it difficult to introduce themselves due to their limited interactions. TaaS does not focus on describing learners' social features, hobbies or resumes, but rather gathering necessary data about learners' individual capabilities. It directly introduces learners by a visual tool, bar chart, thereby establishing a culture of trust within the team.

- The cloud-based jigsaw classroom gives learners opportunities to discuss and understand the different dimensions of team purpose, with the principle that "a better way to learn something is to teach it to someone else". Similarly, they are encouraged to assimilate others' viewpoints.

- Learners participate in real practice to explore the nature of team context, and critically demonstrate how to solve problems. Learners plan for themselves based on their actual situations and skills, thus their tasks are achievable.

- Though challenging, it is essential for team members to pre-plan a way to achieve their target successfully. Detailed task schedules are necessary to avoid confusion and the waste of resources. 
- Learners who see their work as habits rather than choices are more likely to perform better, and have more motivation when faced with difficulties. So we take their preferences into consideration in TaaS.

- [12] suggests that a solution to facilitate collaboration and reduce conflict is that leadership of mobile virtual teams can be shared. We borrow the idea and mend it by abolishing the concentrated leadership and dispersing the duty of it to the both sides of the O2O. Herein, the kind of duty that picks the suited learner to form a capable team and defines the clear-cut role for each team member is in charge of the Inference Service, and the rest duty that monitors team members' work is replaced by mutual supervision among learners.

- We formalize the problem of team grouping into a mathematical task allocation, using SA to achieve the multi-objective optimization that lets learners exploit their talents fully and complement each other's talents. The arbitrariness of team formation is minimized, and some negative interpersonal factors in traditional team learning are avoided.

- Creatively importing peer-assessment in the progress of team learning means that mutual supervision is now available so that learners can keep pace with each other [14]. It promotes positive competition within the team, and decreases the chance that the whole team's outcome be delayed because of a few under-performing members. To some extent, TaaS is also able to detect and prevent a student from claiming another's work as their own.

\section{Conclusion}

In this paper, we introduce a service oriented system, TaaS, to enhance teamwork performance in mobile cloud-based learning. It can work in conjunction with the cloud-hosting LMSs to establish a collaborative learning flow. The five services of TaaS concentrate on covering the gaps caused by the characteristics of mobile environments, making it easy to organize the necessary learner information gathering, efficient discussion, schedule planning, mutual supervision, and rational task allocation. We have implemented TaaS on the Amazon EC2 cloud. In future research, we will bring in case studies to evaluate how much learners' teamwork performance has been enhanced through the use of TaaS.

\section{References}

1. Aronson, E., Blaney, N., Steophan, C., Sikes, J., Snapp, M. : The Jigsaw Classroom. Beverly Hills, CA, USA (1978).

2. Attewell, J.: A Technology Update and m-Learning Project Summary. Mobile Technology and Learning, Learning and Skills Development Agency, UK (2005).

3. Belbin, R.: Team Roles at Work. Butterworth Heinemann (1993).

4. Cao, X. M., Zhao, P., Wang, X.: Study on Design and Development e-Learning System Based on Learning Flow. In: World Congress on Software Engineering (WCSE), (2009). 
5. Chua, F. F., Tay, E. S.: Developing Virtual Learning Environment 2.0 Using Web Services Approach. In: $12^{\text {th }}$ International Conference on Advanced Learning Technologies (2012).

6. Cramton, C. D.: The Mutual Knowledge Problem and Its Consquences for Dispeared Collaboration. In: Organization Science. vol. 12, pp. 346-372 (2001).

7. Feldmann, B.: Group Types in e-Learning Environments-Study Team, Working Team and Learning Team. In: $7^{\text {th }}$ International Conference on Information Technology Based on Higher Education and Trainning (ITHET), Australia (2006).

8. Kayes, A. B., Kayes, D. C., Kolb, D. A.: Developing teams using the Kolb team learning experience. In: Simulation \& Gaming, vol. 36, pp. 355-363 (2005).

9. Kolb, A. D.: Experiential learning: Experience as a source of learning and development. Upper Saddle River, NJ: Prentice Hall (1984).

10. Kolb, A. Y., Kolb, A. D.: Learning styles and learning spaces: Enhancing experiential learning in higher education. In: Academy of Management Learning and Education. vol.4, no.2, pp193-212 (2005).

11. Lingard, $\mathrm{R}$ : Teaching and Assessing Teamwork in Engineering and Computer Science. In: Proceeding of International Symposium on Engineering Education and Educational Technologies(EEET), (2009).

12. Pinola, M. : Mobile Collaboration and Community Tools for Virtual Teams. The Mobile Learning Edge, McGraw-Hill Companies (2011).

13. Rao, N. M.: Cloud Computing Through Mobile-Learning. In: Journal of Computing and Information Technology. vol. 16, no. 4, pp. 235-246 (2008).

14. Sadler, P, M., Good, E.: The Impact of Self- and Peer-grading on Student Learning. In: Educational Assessment. vol. 11, no. 1, pp. 1-31 (2006).

15. Saunders, C. S., Ahuja, M. K: Are All Distributed Teams the Same? Differentiating Between Temporary and Ongoing Distributed Teams. In: Small Group Research. vol.37, no.6, pp.662-700 (2006).

16. Schwabe, G., Goth, C., Frohberg, D.: Does Team Size Matter in Mobile Learning. In: International Conference on Mobile Business (ICMB), Sydney, Australia (2005).

17. Sun, G., Shen, J.: Evaluations of Heuristic Alogrithms for Teamwork-Enhanced Task Allocation in Mobile Cloud-based Learning. In: $17^{\text {th }}$ IEEE International Conference on Computer Supported Cooperative Work in Design (CSCWD).

18. Thiele, J, E.: Learning Patterns of Online Students. In: Journal of Nursing Education. vol. 42, no. 8, pp. 364-367 (2003).

19. Terrell, S, R., Dringus, L.: An Investigation of the Effect of Learning Style on Student Success in an Online Learning Environment. In: Journal of Educational Technology Systems. vol. 28, no. 3, pp. 231-238 (2000).

20. Trifonova, A., Ronchetti, M.: Mobile Learning: Is Anytime + Anywhere = Always Online?. In : $6^{\text {th }}$ IEEE International Conference on Advanced Learning Technologies, Netherlands (2006).

21. Vouk, M. A.: Cloud Computing-Issues, Research and Implementations. In: Journal of Computing and Information Technology, vol. 16, no. 4, pp. 235-246 (2008).

22. Wentling, T. L., Waight, C., Gallaher, J., Fleur, J., Wang, C., Kanfer, A.: e-learning - A Review of Literature. Knowledge and Learning Systems Group, University of Illinois at Urbana-Champaign, Illinois, USA (2000).

23. Wheelan, S. A.: Creating Effective Teams: A Guide for Members and Leaders. Sage Publications (2005).

24. Wu, H., Hamdi, L., Mahe, N.: Tango: A Flexible Mobility-enabled Architecture for Online and Offline Mobile Enterprise Applications. In: $11^{\text {th }}$ International Conference on Mobile Data Management, Kansas, USA (2010). 